\title{
Tres enfoques sobre inteligencia: un estudio con trabajadores manuales
}

\author{
Three focuses on intelligence: A study \\ of manual workers
}

\author{
Daiana Yamila RIGO \\ Danilo Silvio DONOLO'
}

\begin{abstract}
Resumen
Se presenta un estudio de la inteligencia que tiene como objetivo general conocer si los años de experiencia influyen en el modo de ser inteligente de un grupo particular de trabajadores manuales: expertos y novatos. La propuesta se enmarca en tres teorías consolidadas en el campo; nos referimos a la teoría general de la inteligencia, la teoría de las inteligencias múltiples y la teoría triárquica de la inteligencia. La investigación se realiza con 149 trabajadores manuales, a los cuales se les administra el RAVEN, el Multiple Intelligences Developmental Assessment Scale y el Sternberg Triarchic Abilities Test. Los resultados muestran diferencias significativas en las inteligencias presentes en el grupo cuando se lo estudia en función a los años de experiencia: expertos y novatos. Se concluye que tal variable está relacionada con el contexto laboral por cuanto delimita las formas de inteligencias que se van desarrollando conforme se adquiere experiencia en el campo práctico de la profesión.
\end{abstract}

Unitermos: Inteligencias; Personal; Interpretación teórica.

\begin{abstract}
We present a study of intelligence with the aim of knowing ifyears of experience influence the way of intelligence among a particular group of manual workers: experts and novices. The proposal is framed within three consolidated theories in the field; we refer to the general theory of intelligence, the multiple intelligences theory and the triarchic theory of intelligence. The research is carried out using 149 manual workers, who are given the RAVEN, the Multiple Intelligences Developmental Assessment Scale and the Sternberg Triarchic Abilities Test tests. The results show significant differences in the intelligence present in the group when studied on the basis of years of experience: experts and novices. We conclude that this variable is related to work context which delimits the forms of intelligence that are developed while acquiring experience in the practical area of the profession.
\end{abstract}

Uniterms: Intelligence; Manual workers; Theoretical perspectives.

\section{Diversas teorías sobre la inteligencia}

Uno de los problemas más acuciantes cuando se adentra al campo de la inteligencia es la multiplicidad de perspectivas teóricas desarrolladas y la complejidad que adquiere el concepto por la cantidad de significados que se le asignan. De manera análoga, se observa en la definición de los estudios; un posicionamiento cerrado a determinadas concepciones. Al respecto, Sternberg (1985a) advirtió, hace algunos años, un clima tenso entre las teorías, aún vigentes, expresado en un reclamo por la

$\operatorname{crt}$

1 Universidad Nacional de Río Cuarto, Faculta de Ciencias Humanas, Departamento Ciencias de la Educacion. Rieta Nascional, 36, KM 601, Rio Cuanto, Cordoba, Argentina. Correspondência para/Correspondence to: D. Y. RIGO. E-mail: <daianarigo@hotmail.com>. 
integración de enfoques para entender el complejo entramado que supone ser inteligente.

En respuesta, la perspectiva de múltiples formas de ser inteligente ha ido tomando relevancia en el campo de la investigación y conformando nuevas líneas de estudio que tienen como propósito conocer cómo las personas se muestran inteligentes en sus contextos de práctica (Gardner, 1983; Rigo \& Donolo, 2012; Robinson \& Aronica, 2010; Sibilia, 2005; Sternberg, 1985b). En este sentido, se entiende que las habilidades cognitivas que usa un científico difieren de las usadas por un educador, a la vez que las tareas y actividades que definen a cada campo son propias de cada disciplina.

En el marco formulado sobre diversos modos de ser inteligente, proponemos un estudio de la inteligencia desde tres teorías consolidadas teórica y empíricamente, que se fundamenta sólo en la necesidad de comprender la complejidad de la inteligencia en sus diversas manifestaciones, sino también en estudiar el modo de ser inteligente en un grupo particular de trabajadores manuales, atendiendo a los años de experiencia en el continuo novato-experto, para entender cómo la práctica media en las habilidades cognitivas presentes en la muestra propuesta.

Por tanto, se entiende que estudiar la inteligencia desde una postura teórica limita el foco a algunas habilidades cognitivas, descuidando la atención a otras igualmente importantes, de manera tal que se consideren tres teorías para el presente estudio, a saber: general, múltiple y triárquica, como una alternativa para entender e investigar los diversos modos de ser inteligente. Seguidamente, se definen las perspectivas teóricas que interesan.

\section{Inteligencia general}

La teoría del factor $g$ formulada a inicios del siglo XX de la mano de Spearman tuvo y tiene una gran influencia en el campo de la inteligencia, no sólo en su formulación inicial, sino también en las perspectivas más actuales que la integran, como las propuestas de Carroll (1993) y de Jensen (1999) que han demostrado su existencia y su integración en el marco de un modelo jerárquico de la inteligencia, que va mucho más allá de la teoría bifactorial propuesta por el propio Spearman en 1927 (Colom y Andrés-Pueyo, 1999). Una perspectiva que define a la inteligencia como una capacidad individual cuya manifestación se da en diferentes tipos de situaciones y problemas, para cuya resolución se ponen en juego habilidades lógicas y abstractas, tales como las de educir relaciones y correlatos.

Asimismo, su importancia y permanencia en el campo y el pleno interés en su investigación, la han convertido en un asunto de estudio interesante que pasa a formar parte de los journals más específicos que existen en torno a la inteligencia, destacándose entre ellos intelligence, que reporta algo más de $2.399 .953^{2}$ artículos para la expresión general factor. De hecho, Neisser et al. (1996) lo confirma al exponer que las aproximaciones a la inteligencia desde la psicometría, no sólo han inspirado parte de la investigación y atraído la mayor parte de la atención, sino que además esta última es la más empleada en la práctica.

Los autores que apoyan la teoría del factor general también rescatan esta tendencia, para quienes no es sorpresa su vigencia actual. Jensen (1999) expresa que sólo basta constatar su validez al comprobar su lugar de preeminencia en los modelos factoriales de inteligencia más aceptados en el presente, bien como un factor de tercer orden en los modelos jerárquicos, 0 bien identificado con un factor de segundo orden en el modelo de Cattell.

Más recientemente, los postulados sobre $g$ se ven respaldados por el desarrollo de las neurociencias, que buscan estudiar, como Neisser et al. (1996) advierte en Intelligence: Knowns and Unknowns, el cerebro como base para explicar qué es la inteligencia y cómo medirla. Más específicamente, se estudia qué región del cerebro corresponde al funcionamiento inteligente. En general, los resultados de diversas investigaciones apuntan a confirmar la Teoría de la Integración Parieto-Frontal (Haier \& Jung, 2010; Svitil, 2010).

En suma, la teoría del factor general ha recibido y tiene aún un gran apoyo en el campo de la psicología de la inteligencia, y por ello la importancia de considerarla en investigaciones actuales como una

402 Consultado, 5-mar-2012, en ScienceDirect. 
alternativa, entre otras, para seguir entendiendo el constructo en cuestión.

\section{Inteligencias múltiples}

Una consideración más reciente, formulada a finales de siglo XX, propone un fundamento múltiple para entender la inteligencia. Se trata de la teoría de las inteligencias múltiples desarrollada por Gardner en 1983, en la cual se plantean tres grupos de perfiles intelectuales: académico: inteligencia lingüística - la capacidad para usar las palabras de manera efectiva, sea de manera oral o de manera escrita - y lógica - matemática - la habilidad para utilizar los números de manera efectiva y razonar adecuadamente -; artístico: inteligencia espacial - la capacidad para percibir el mundo visual-espacial y de ejecutar transformaciones sobre esas percepciones -, cinestésica - la capacidad para usar todo el cuerpo para expresar ideas y sentimientos, así como la facilidad en el uso de las propias manos para producir o transformar cosas- y musical - la habilidad de percibir, discriminar, transformary expresar las formas musicales -; emocional: interpersonal - la capacidad de percibir y establecer distinciones en los estados de ánimo, las intenciones, las motivaciones y los sentimientos de otras personas e intrapersonal - el conocimiento de sí mismo y la habilidad para adaptar las propias maneras de actuar a partir de ese conocimiento -; más recientemente se integra un cuarto grupo con la denominada inteligencia naturalista - la capacidad de reconocer y de clasificar numerosas especies, así como la habilidad para distinguir y categorizar organismos nuevos o poco familiares (Gardner, 2001).

Sus postulados principales y sobre los cuales se han enfocado los desarrollos teóricos y prácticos, en parte, buscan entender las relaciones entre inteligencias y contextos. En este sentido, Gardner (2004) expresa la importancia de observar que tanto Wolfgang Amadeus Mozart, con sus 626 composiciones catalogadas por Köchel, como Martha Graham, con sus docenas de coreografías, o Pablo Picasso, con sus miles de pinturas y dibujos, no llevaron a cabo el mismo conjunto de operaciones lógicas que un físico o un matemático, sino más bien se comprende que las operaciones mentales que se ponen en juego cuando pintamos, cantamos o resolvemos un enigma matemático son diferentes.
Estas formulaciones se sustentan en su definición de inteligencia como un potencial biopsicológico para procesar información, la cual puede ser activada dentro de un marco cultural para resolver problemas o crear productos que tienen valor para una cultura (Gardner, 2001); es decir, cada perfil intelectual comprende, por un lado, el dominio de las habilidades necesarias para la resolución de problemas, y, por otro lado, las habilidades para encontrar o plantear nuevos problemas. A su vez, este perfil cambia en situaciones particulares; de hecho, la importancia de cada inteligencia radica en la utilidad que adquiere en determinados ambientes culturales y sociales.

Asimismo, el tema de las inteligencias múltiples es sugerente para comprender la diversidad de manifestaciones que se pueden encontrar en los diferentes dominios culturales, organizados a través de tareas, sistemas simbólicos y un conjunto de operaciones donde se hacen manifiestas las interrelaciones entre inteligencias (Nikolova \& Taneva-Shopova, 2007). Por lo tanto, esta perspectiva rescata que no existe una correspondencia lineal entre domino e inteligencias sino que algunas de ellas se muestran más apropiadas a los fines de uno u otro campo, y por ello se habla de habilidades amalgamadas más que aisladas en la pericia de un ámbito.

En general, la importancia de la teoría en las investigaciones actuales, y específicamente en el estudio de expertos y novatos, está en la posibilidad de estudiar si las personas con marcada experiencia en un campo se expresa de los estados finales de las inteligencias y por tanto muestran un perfil más marcado en las inteligencias que están más relaciones con las actividades desempeñadas.

\section{Inteligencia triárquica}

Otra teoría que se retoma en el estudio es la postulada por Sternberg (1985b), que brinda una alternativa para comprender las habilidades que los sujetos ponen en funcionamiento para la resolución de los problemas. La propuesta de la teoría triárquica que nos interesa mantiene vigencia en consideraciones teóricas más recientes por parte del autor y de otros investigadores, con implicancias prácticas en la medición de la inteligencia (Stemler, Sternberg, Grigorenko, Jowin \& Sharpes, 2009; Sternberg, 2009). Además, es una 
propuesta que entiende que la capacidad del ser humano se extiende más allá del razonamiento analítico, y en donde la creatividad y el conocimiento tácito se consideran recursos cognitivos igualmente válidos en el contexto de las experiencias actuales.

De esta forma, la teoría entiende que los sujetos cuentan con una amplia variedad de herramientas y estrategias que se orientan de cara a diversos contextos y condiciones (Rigo \& Donolo, 2010;2011). A decir verdad, no asombra ver que dos sujetos actúen de modo muy distinto frente a la necesidad de resolver una situación idéntica. En este sentido, desde una perspectiva triárquica, Sternberg (1985b) postula tres tipos de inteligencias que las denomina como creativa, práctica y analítica.

La inteligencia creativa es la capacidad para ir más allá de lo dado y engendrar ideas nuevas e interesantes. Asociada al pensamiento sintético, son los sujetos que crean las conexiones con el conocimiento, el cual a su vez pertenece a diferentes campos disciplinarios o contextos, que no son percibidos por otras personas; igualmente crean nuevas soluciones para problemas novedosos. La creatividad, asimismo, se relaciona con la innovación, lo novedoso, lo original, el inconformismo con lo disponible, la genialidad, el descubrimiento; en definitiva, con lo no conocido (Sternberg, 2000; Sternberg \& Lubart, 1997). La postura de Sternberg es interesante por cuanto asocia el componente de creatividad a la inteligencia, mostrando una perspectiva constitutiva y no complementaria a la misma.

Por otro lado, la inteligencia práctica es la capacidad para transportar la teoría a la práctica, lo cual conlleva la aplicación, uso, implementación y puesta en práctica de las ideas. En general, las investigaciones han centrado los estudios en uno de los aspectos de la inteligencia práctica, el conocimiento tácito, definido como un saber implícito que se aprende a través de la experiencia en contextos particulares de actividades genuinas. Polanyi (1966) postula que se puede saber más de lo que se puede decir, aludiendo así a un saber asociado con el hacer que se caracteriza por un alto nivel de habilidad relacionado a la poca capacidad para la deliberación o la atención enfocada.

Por último, la inteligencia analítica es la 42 capacidad para analizar y evaluar ideas, revolver problemas y tomar decisiones. Más específicamente, está implicada cuando se emplean los componentes del procesamiento de la información en la comparación, contraste y valoración de los pensamientos, en una consideración de problemas que se definen por ser familiares, poco novedosos, de naturaleza fundamentalmente abstracta y de formato estructurado y bien definido (Sternberg, 1985b).

En general, la propuesta de la teoría triárquica confiere un lugar muy considerado a las manifestaciones creativas y a los entornos en los que puede manifestarse, ofreciendo a su vez una alternativa para comprender cómo diferentes personas en contextos disímiles pueden hacer uso de diversas modalidades para la resolución de problemas. Asimismo, es interesante estudiar las relaciones de inteligencia entre expertos y novatos, por cuanto se considera que las habilidades lógicas tienden a decrecer con la edad y crece la inteligencia práctica ese modo de saber hacer que se desprende del saber que está más relacionado con la inteligencia analítica o general -, tal cual lo confirman los estudios de Gottfredson (2002) y Sternberg (2000).

Ahora bien, en la consideración de las tres teorías descritas, conocer el tipo de inteligencia presente en los trabajadores manuales es esencial para crear zonas para la aparición, el mantenimiento y el desarrollo de experiencias que estén asociadas con desempeños más ajustados a los contextos reales. De igual manera, se brinda una base para entender las habilidades cognitivas que se asocian a campos laborales particulares, visualizando la existencia de habilidades específicas relacionadas con los diferentes dominios de trabajo y estudio. En este sentido, los especialistas en el tema entienden que las habilidades entre expertos y novatos varían en función de cómo organizan, representan e interpretan la información (Bransford, Brown \& Cocking, 2000), con lo cual se vuelve crucial conocer los modos de ser inteligente para educar en consecuencia, entendiendo que la perspectiva de diversas teorías sobre la inteligencia puede ofrecer indicios para su comprensión. Asimismo, las investigaciones previas indican que cuando se estudia a novatos y expertos es posible encontrar diferencias en el uso de un conocimiento más declarativo o procedimental (Chi \& Glaser, 1980); por otro lado, Yarlas y Sloutsky (2000) denotan diferencias en la forma de clasificar las ecuaciones matemáticas haciendo uso de estructuras más superficiales o más profundas y, de la misma forma, 
Ryle (1967) destaca que las diferencias entre ambos grupos se encuentra entre el saber cómo y el saber qué. Estos antecedentes nos permiten suponer que las inteligencias pueden variar en sujetos que tienen mayor o menor experiencia en el campo de las labores manuales.

Finalmente, a partir de las tres teorías definidas y contando con una propuesta en un contexto particular, se describe a continuación el estudio realizado, el objetivo del mismo y los resultados hallados.

El objetivo del estudio es conocer los modos de ser inteligente; en términos de las habilidades cognitivas que definen a un grupo de trabajadores manuales, buscando conocer cómo la variable de años de experiencia, definida en el continuo novato-experto, se relaciona con el desarrollo de determinadas inteligencias definidas en el interior de la teoría general, múltiple y triárquica de la inteligencia.

\section{Método}

\section{Participantes}

La investigación fue desarrollada en el año 2011 en la ciudad de Río Cuarto (Córdoba, Argentina), de la que participaron 149 trabajadores manuales. El grupo, en general, presenta características que varían en función de los años de experiencia en actividades manuales. Entre este tipo de trabajos realizados por los colaboradores se encuentran los siguientes oficios: albañilería, carpintería, peluquería, costura, mecánica, repostería, pedicuría, cocina, electricidad, pintura, decoración, panadería y plomería.

Respecto a la dimensión años de experiencia en el desempeño de la profesión, encontramos en general para la muestra un valor mínimo de 1 año y un valor máximo de 40 años. Por tal razón se construyeron dos categorías, considerándose la mediana (los sujetos que tenían 6 años de experiencia o menos pasaron a formar el grupo de novatos; entretanto, los trabajadores con experiencia entre 7 y 40 años formaron el grupo de los expertos) como punto de corte, a fin de reagrupar a los sujetos. Por tanto, la muestra se dividió entre los que llevan menos tiempo ejerciendo oficios de tipo manual y aquellos que llevan más tiempo realizando actividades manuales, para estudiar así sus habilidades cognitivas en función de la variable años de experiencia, quedando definido como se muestra en la Tabla 1. Es decir, se eligió ese criterio para la creación de ambos grupos, por cuanto se considera que la distribución final es homogénea además de ser una medida robusta, lo cual permite estudiar si la variable años de experiencia influye en las inteligencias presentes en los trabajadores manuales.

De esta forma, el grupo de novatos quedó conformado por 75 sujetos con una edad media de 26 años; del total, 48 eran mujeres y el resto varones. El grupo de expertos quedó integrado por 74 personas con una edad promedio de 44 años, de las cuales 38 eran mujeres y 36 varones.

\section{Instrumentos}

Los instrumentos seleccionados para este trabajo son tres: el RAVEN, el Multiple Intelligences Developmental Assessment Scale (MIDAS) y el Sternberg Triarchic Abilities Test (STAT).

El test de matrices progresivas, conocido generalmente como test de Raven (1971) y el cual mide el factor general de la inteligencia, se utiliza para evaluar la capacidad para la comprensión y el manejo mental de las relaciones abstractas y el pensamiento lógico a través de problemas en los hay que completar figuras geométricas abstractas y lacunarias dentro de un sistema de relaciones de complejidad creciente. El test está integrado por 5 series: A, B, C, D, E, cada una compuesta por 12 problemas que conforman un total de 60.

El instrumento Escala Evolutiva de Evaluación de Inteligencias Múltiples (MIDAS-A) de Shearer (2007) se emplea con el objetivo de conocer los perfiles intelectuales y es una de las propuestas más interesantes y consolidadas de medición de las IM. A través de ocho

\section{Tabla 1}

Características generales por años de experiencia: novatos y expertos. Río Cuarto, 2011

\begin{tabular}{lcc}
\hline Características de la muestra & Novatos & Expertos \\
\hline$N$ & 75 & 74 \\
Edad Media & 26 & 44 \\
Género & & \\
Feminino & 48 & 38 \\
Masculino & 27 & 36 \\
Años de experiencia & 1 a 6 & 7 a 40 \\
\hline
\end{tabular}


escalas, el cuestionario indaga las inteligencias múltiples propuestas por Gardner (1983), con formato de respuesta múltiple choice. El MIDAS está compuesto por 119 ítems que brindan información del grado en el que la habilidad consultada se presenta en el desarrollo personal de cada sujeto.

Por último, el STAT, el test de habilidades triárquicas de Sternberg (1993) y único test desarrollado para medir la propuesta triárquica, se emplea con el objetivo de valorar las habilidades analíticas, prácticas y creativas. El instrumento consta de 36 problemas que se distribuyen en tres escalas: analítica, práctica y creativa, que a su vez están integradas por tres dominios: verbal, numérico y figurativo, con un total de 12 problemas por escalas y 4 por dominio.

Para cada instrumento se calculó el alpha de Cronbach a fin de conocer su fiabilidad. Para el RAVEN, éste fue de 0.84; para las ocho escalas del MIDAS los índices oscilaron entre 0.80 y 0.84 ; y para las tres dimensiones del STAT se encontraron valores de 0.84 . Estos resultados sugieren una confiabilidad aceptable para el instrumento y sus respectivas escalas. Asimismo, se estimaron las correlaciones entre las escalas, encontrándose valores moderados, no superiores a 0.50, lo que indicaría, en general, una independencia entre las inteligencias medidas por cada instrumento.

\section{Procedimiento}

En cuanto a la administración, se llevó a cabo en los lugares de trabajo de los sujetos valorados y se optó por la modalidad individual. Los instrumentos RAVEN y STAT se aplicaron en dos sesiones en formato impreso. En la primera, se solicitó que se completara el test de Matrices Progresivas de Raven con un promedio de tiempo de 45 minutos, y se administró parte del STAT en lo referente a las secciones 1 a 3 que miden la inteligencia analítica. En la segunda se administraron las secciones 4 a 9 del STAT, que miden la inteligencia práctica y la creativa. Las instrucciones fueron brindadas por instructor entrenado en las características de ambos tests.

Para el MIDAS se escogió la modalidad autoadministración. Así, el cuestionario fue entregado al sujeto en valoración, a quien se explicó la consigna y la modalidad para completar la hoja de respuesta,

44 entregándole inmediatamente después el material impreso (cuadernillo, hoja de respuesta y consigna). El mismo fue completado en el intervalo de las dos secciones programadas para la administración del RAVEN y el STAT, tomando alrededor de 45 minutos para rellenarlo.

En cada caso se solicitó el consentimiento de los participantes, informándoles sobre los objetivos de la investigación, la modalidad de la misma, sus propósitos, el por qué se les seleccionó para participar en ella, cuánto tiempo y esfuerzo requeriría su participación, quién tendría acceso a los resultados y cómo se usarían los datos en el futuro, asegurando siempre la confidencialidad de los datos y el anonimato de estos.

\section{Resultados}

Conforme a los análisis realizados, se presentan los resultados derivados del estudio descriptivo y diferencial en relación con la variable años de experiencia novato-experto en función a cada teoría.

Como se muestra en la Tabla 2, observamos que el grupo de novatos y expertos difieren en algunos valores medios que podrían estar ofreciendo algún indicio de cómo las habilidades de los trabajadores manuales se desarrollan en función de los años de experiencia.

\section{Tabla 2}

Media (M) y desviación estándar (SD) para las inteligencias valoradas por el MIDAS, RAVEN y STAT en novatos y expertos

\begin{tabular}{lccccc}
\hline \multirow{2}{*}{ Inteligencia } & \multicolumn{2}{c}{ Novato } & & \multicolumn{2}{c}{ Experto } \\
\cline { 2 - 3 } \cline { 5 - 6 } \cline { 5 - 6 } MIDAS & & SD & & $M$ & SD \\
Musical & 38,24 & 15,93 & & 32,28 & 14,27 \\
Cinestésica & 45,49 & 14,59 & & 42,42 & 16,67 \\
Lógica-Matemática & 42,20 & 12,96 & & 41,86 & 13,65 \\
Espacial & 43,41 & 14,78 & & 50,22 & 18,2 \\
Lingüística & 44,51 & 15,68 & & 41,85 & 15,7 \\
Interpersonal & 52,69 & 14,97 & & 54,04 & 14,77 \\
Intrapersonal & 47,85 & 12,68 & & 53,34 & 13,54 \\
Naturalista & 40,93 & 17,35 & & 47,73 & 15,22 \\
RAVEN & & & & \\
General & 49,36 & 6,17 & & 45,77 & 6,81 \\
STAT & & & & \\
Analítica & 5,89 & 2,18 & 4,16 & 2,32 \\
Práctica & 4,33 & 2,33 & 7,53 & 2,41 \\
Creativa & 6,03 & 2,78 & 5,84 & 2,35 \\
\hline
\end{tabular}


Para conocer si tales diferencias eran significativas se procedió a realizar una prueba $t$ con muestras independientes, a partir de la cual se encontró que el grupo de expertos presenta un mayor dominio de las habilidades espaciales $(t=-2,506 ; p=0,013)$, intrapersonales $(t=-2,552 ; p=0,012)$ y naturalistas $(t=-$ $2,541 ; p=0,012)$ valoradas a través del MIDAS y en inteligencia práctica por el STAT ( $t=-8,227 ; p=0,000)$. Por su parte, el grupo de novatos mostró una mayor apertura en la inteligencia musical $(t=2,403 ; p=0,018)$ del MIDAS, mayores manejos de las relaciones lógicas en el RAVEN ( $t=3,374 ; p=0,001)$ y mejor capacidad de resolución de problemas analíticos $(t=4,686 ; p=0,000)$ en el STAT. Por otro lado, no se hallaron diferencias significativas para las inteligencias cinestésica, lógicamatemática, lingüística e interpersonal, para las cuales los valores medios de ambos grupos fueron cercanos, al igual que en la inteligencia creativa del STAT.

Asimismo, y considerando los valores de la desviación estándar, se entiende que los modos de ser inteligente, si bien presentan cierta homogeneidad en cuanto a perfiles más marcados en algunas inteligencias para expertos y novatos, también son idiosincrásicos a cada sujeto que compone la muestra, dada su dispersión. Dicha variabilidad se puede observar en los siguientes diagramas de caja, donde cada uno muestra las inteligencias valoradas por el MIDAS, el RAVEN y el STAT y en las cuales se hallaron diferencias significativas entre novatos y expertos. Cada diagrama de caja indica los valores que se sitúan entre el primer y el tercer cuartil; la mediana está representada por una línea que divide la caja en dos partes. las cuatro dimensiones valoradas por el MIDAS, se aprecia una tendencia que apoya en principio a los datos ofrecidos en la Tabla 2 e indicarían diferencias moderadas para la inteligencia musical entre ambos grupos, con mayor dispersión en el grupo de expertos entre los valores mínimo y máximo; para la inteligencia espacial, la Figura 1B muestra más simetría para el grupo de expertos y una diferencia respecto al grupo de novatos, para el cual se observa una mayor dispersión para las puntuaciones por debajo de la mediana. En la Figura 1C se observa una distribución más asimétrica para el grupo de novatos, con una clara tendencia a favor del grupo de expertos para la inteligencia intrapersonal. En relación con la Figura 1D, ambas distribuciones se muestran simétricas en inteligencia naturalista, con una leve diferencia entre los grupos y mayor dispersión de los datos para los novatos.

Respecto a las inteligencias valoradas por el STAT, los diagramas de caja Figura 2A y 2B muestran diferencias evidentes para ambos grupos con distribuciones asimétricas, una tendencia más práctica

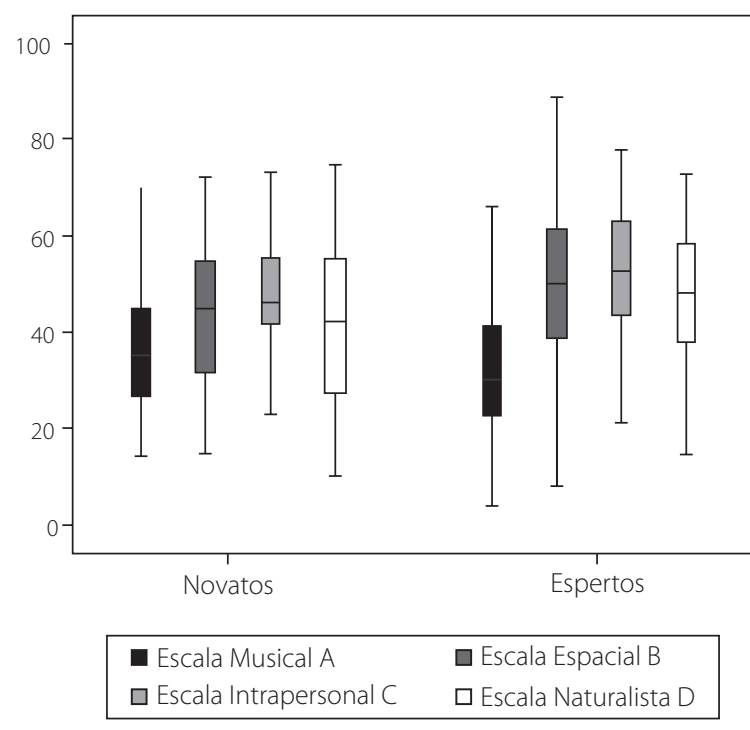

Figura 1. Diagrama de la caja de puntajes en inteligencia musical (A), espacial (B), intrapersonal y naturalista (D) para trabajadores manuales, novatos y expertos.

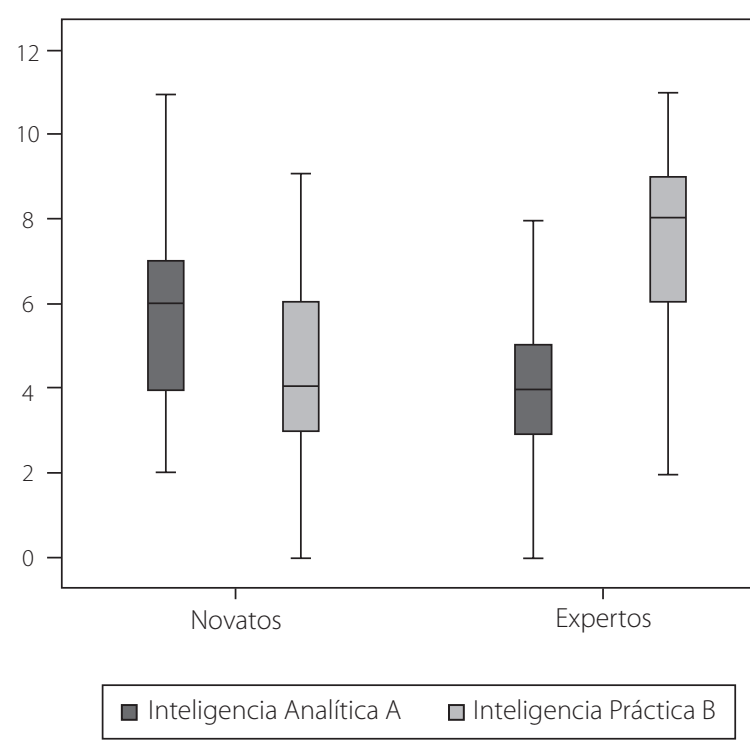

Figura 2. Diagrama de la caja de puntaje en inteligencia práctica (A) y analítica (B) para trabajadores manuales, novatos y expertos. 


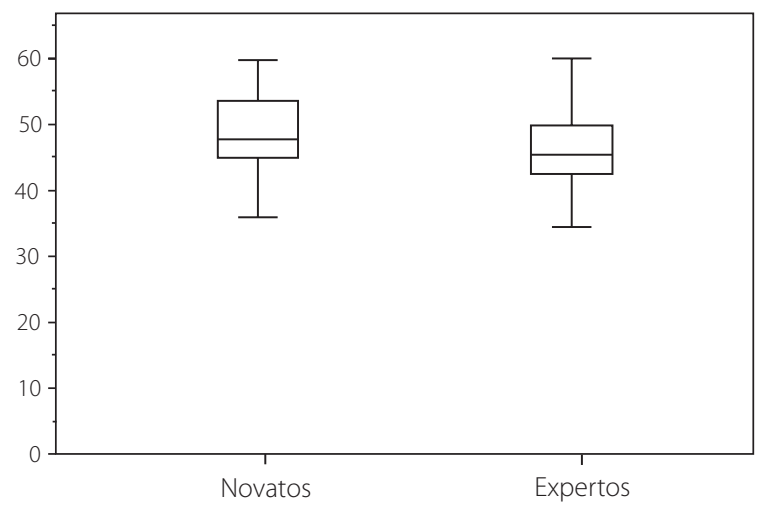

Figura 3. Diagrama de la caja de puntajes en inteligencia general para trabajadores manuales, novatos y expertos.

en el grupo de expertos y más analítica en los trabajadores manuales novatos.

Por último, se aprecia en la Figura 3, y confirmando los datos de la Tabla 2, que el grupo de novatos presenta una inclinación marcada al pensamiento lógico y abstracto medido por el RAVEN, con mayor dispersión en las puntuaciones comprendidas por encima de la mediana.

\section{Discusión y Conclusión}

La presente investigación tuvo como objetivo conocer los modos de ser inteligente de un grupo particular de trabajadores manuales respecto a la dimensión experto-novato. Además se consideraron, para su estudio, tres teorías consolidadas teoricamente y empíricamente en el campo de la inteligencia: la inteligencia general, múltiple y triárquica, así como sus respectivos instrumentos RAVEN, MIDAS y STAT. EI estudio comenzó considerando que si se estudiaba la inteligencia en función de los años de experiencia, se podría comprender más a fondo cuáles son las habilidades que usan los novatos y expertos cuando el oficio en cuestión se refiere a lo manual. Asimismo, se eligió para su estudio una perspectiva múltiple considerando diferentes teorías y medidas, a fin de ofrecer una visión compleja de las habilidades cognitivas de los sujetos. Se entiende que esta postura facilita una lectura más compleja del constructo en estudio, y decisiones más ajustadas para transferir los resultados

46 a planes de formación para el trabajo.
Respecto a los resultados, y en función de las tres perspectivas consideradas, entendemos que las inteligencias en trabajadores manuales expertos y novatos varían en diversos aspectos, apoyando así la relación año de experiencia y habilidades cognitivas. En este sentido, observamos que el grupo con mayor experiencia cuenta con un mayor manejo espacial una de las inteligencias que más aportan al quehacer laboral -, lo cual coincide con las investigaciones y los estudios de Gardner (2001) y de Shearer (2007) cuando mencionan que el manejo de las relaciones espaciales, de diseño, diagramación y modelado de un objeto es afín al campo de lo manual. Asimismo, el grupo de expertos presenta mayor dominio intrapersonal y naturalista, inteligencias que por su naturaleza entendemos que podrían contribuir de manera secundaria a la definición de inteligencia en el área de los trabajadores manuales, al igual que el perfil musical del grupo de novatos. Por tanto, la capacidad para diseñar, dibujar, construir y crear que define al perfil espacial parece indicar que son éstas las habilidades que más se desarrollan con la experiencia en el área manual, aunque también se esperaba encontrar un perfil elevado en cinestésica en la delimitación de este tipo de trabajo por su relación con los movimientos finos y gruesos.

Por otro lado, y en lo referente a la relación entre conocimiento lógico y tácito, se encontró que los trabajadores que llevan más años haciendo la tarea demuestran tener mayor destreza en el área de la inteligencia práctica; mientras que, por el contrario, los que recién se están iniciando en lo manual parecen contar con habilidades lógicas y analíticas tanto en el RAVEN como en el STAT. Así, los resultados se sitúan en la línea de lo planteado en diversos estudios sustentados en la teoría triárquica de las inteligencias, en cuanto se fortalece la tendencia que autores como Neisser et al. (1996), Gottfredson (2002; 2003), y Sternberg (2000) postulan acerca de las capacidades más académicas cuando expresan que éstas tienen un desarrollo más marcado en edades más tempranas, tendiendo a disminuir luego de la adultez. Además, así como hay una mayor presencia de habilidades académicas en el grupo de novatos, encontramos que los trabajadores manuales con mayor experiencia en el campo profesional muestran superior conocimiento tácito. Esto denota al menos un aspecto de importancia para 
comprender el desarrollo de habilidades específicas al contexto laboral-manual. Es decir, que el desarrollo de la experticia en las actividades propias a la profesión, conforme se suman años de experiencia en su realización, se asocia a un mayor conocimiento tácito y a una disminución de la inteligencia general. Esta tendencia ha sido confirmada por algunos estudios de Hedlun, Antonakis y Sternberg (2002), y Sternberg y Grigorenko (2001), quienes indican que el sentido común soporta los recursos cognitivos que se ponen en juego para tomar decisiones y plantear alternativas para la solución de problemas cuando se tiene mayor experiencia en el campo.

En suma, en función de los resultados encontrados creemos que se tienen algunas pistas para empezar a pensar en diversos modos de ser inteligentes en función de la experiencia transcurrida en trabajos relacionados con lo manual; es decir, los datos de esta investigación informan que las habilidades de expertos y novatos responden a diversos modos de organizar, representar e interpretar la información para resolver problemas y hacer frente a las tareas que se desempeñan con particularidad en cada sujeto. Asimismo, consideramos que el estudio realizado contribuye a pensar en la formación profesional y laboral en relación a las inteligencias que se deben potenciar y acerca de cómo organizar la educación técnica con un enfoque orientado a la práctica, centrado más en el saber cómo, más que en el saber qué, encaminado al desarrollo de los conocimientos tácitos y las habilidades espaciales. Por último, también encontramos que, en futuras investigaciones, es importante seguir indagando las inteligencias en otros contextos, con otras muestras y otros instrumentos de recolección de datos, de modo a ampliar el alcance de lo hallado y especificar acciones más concretas. De esa forma podrán extenderse las posibilidades de generalizar los resultados a partir de muestras probabilísticas, por cuanto se pueden desarrollar versiones reducidas y adaptadas de los instrumentos aplicados y sortear dos limitaciones del estudio presentado: el tiempo de aplicación y el tamaño de la muestra.

\section{Referencias}

Bransford, J., Brown, A., \& Cocking, R. (Ed.). (2000). How people learn: Brain, mind, experience and school. New York: Nacional Academy Press.
Carroll, J. B. (1993). Human cognitive abilities: A survey offactoranalytical studies. New York: Cambridge University Press.

Chi, M., \& Glaser, R. (1980). The measurement of expertise: Analysis of the development of knowledge and skill as a basis for assessing achievement. In E. Baker \& E. Quellmalz (Eds.), Educational festing and evaluation: Design, analysis and policy (pp.37-47). Beverly Hills, CA: SAGE.

Colom, R., \& Andrés-Pueyo, A. (1999). El estudio de la inteligencia humana: recapitulación ante el cambio de milenio. Psicothema, 11(3), 453-476.

Gardner, H. (1983). Frames of mind: The theory of multiples intelligences. New York: Basic.

Gardner, H. (2001). La inteligencia reformulada: las inteligencias múltiples en el siglo XXI. Barcelona: Paidós.

Gardner, H. (2004). Mentes flexibles: el arte y la ciencia de saber cambiarnuestra opinión y la delos demás. Barcelona: Paidós.

Gottfredson, L. (2002). Where and why $g$ matters: Not a mystery. Human Performance, 15(1/2), 25-46.

Gottfredson, L. (2003). g, jobs and life. In H. Nyborg (Ed.), The scientific study of general intelligence: Tribute to Arthur R. Jensen (pp.293-342). Oxford: Elservier.

Haier, R., \& Jung, R. (2010). Identifican la red cerebral vinculada a la inteligencia. Noticia publicada en Sólo Ciencias. Recuperado el agosto 27, 2012, en <http://www. solociencia.com/medicina/07101801.htm>.

Hedlund, J., Antonakis, J., \& Sternberg, R. (2002). Tacit know/wdge and prcatical intelligence: Understanding the lessons of experience. Retrieved August 29, 2012, from $<$ http://www.au.af.mil/au/awc/awcgate/army/ari_tacit_ knowledge.pdf>.

Jensen, A. (1999). The G factor: The science of mental ability: revisión de libro por Secades, R. Psicothema, 11(2), 445-446.

Neisser, U., Boodoo, G., Bouchard, T., Boykin, N., Ceci, S., \& Halpern, D., et al. (1996). Intelligence: Knowns and unknowns. American Psychologist, 51(2), 77-101.

Nikolova, K., \& Taneva-Shopova, S. (2007). Multiple Intelligence theory and educational practice. Annual Assesn Zlatarov University, 26(2), 105-109.

Polanyi, M. (1966). The tacit dimension. New York: Doubleday \& Company.

Raven, J. (1971). Test de matrices progresivas para la medida de la capacidad mental: escala general. Manual. Buenos Aires: Paidós.

Rigo, D., \& Donolo, D. (2010). Una medida de las inteligencias múltiples en contextos universitarios. REDEC, 6(2), 1-11.

Rigo, D., \& Donolo, D. (2011). Inteligencia triárquica: cuando el pensar adopta múltiples modos. Revista Cognición, 7(35), 1-11

Rigo, D., \& Donolo, D. (2012). ¿De qué modo somos inteligentes? Resultados para pensar la educación! Revista Cultura y Educación, 24(1), 5-15.

Robinson, K., \& Aronica, L. (2010). El elemento: descubrir tu pasión lo cambio todo. Grijalbo: Buenos Aires. 
Ryle, G. (1967). El concepto de lo mental. Paidós: Buenos Aires.

Shearer, B. (2007). The MIDAS: A professional manual. Kent, Ohio: Research and Consulting.

Sibilia, M. (2005). Tecnología educativa constructivistahumanista. REICE, Eficacia y Cambio en Educación, 3(1), 799-816.

Stemler, S., Sternberg, R., Grigorenko, E., Jarvin, L., \& Sharpes, K. (2009). Using the theory of successful intelligence as a framework for developing assessments in AP physics. Contemporany Educational Psychology, 34(3), 195-209.

Sternberg, R. (2000). Inteligencia exitosa: cómo una inteligencia práctica y creativa determinan éxito en la vida. Buenos Aires: Paidós.

Sternberg, R. (1985a). Beyond IQ: A triarchic theory of human inelligence. New York: Cabridge University Press.

Sternberg, R. (1985b). The triarchic mind. New York: Penguin Books.

Sternberg, R. (1993). Sternberg triarchic abilities Test - STAT. (Unpublisherd test).

Sternberg, R. (2009). Assessment of gifted students for identification purposes: New techniques for a new millennium. Learning and Individual Differences, 20(4), 327-336.

Sternberg. R., \& Grigorenko, E. (2001). Practical intelligence and the principal. Retrieved August 29, 2012, from <http:// www.temple.edu/Iss/pdf/publications/pubs20012.pdf $>$.

Sternberg, R., \& Lubart, T. (1997). La creatividad en una cultura conformista: un desafío a las masas. Barcelona: Paidós.

Svitil, K. (2010). La conexión entre ciertas regiones cerebrales determinan la inteligencia general. Recuperado el agosto 29, 2012, en <http://www.noticias21.com/node/2309>.

Yarlas, A., \& Sloutsky, V. (2000). Problem representation en experts and novices: Part 1. Differences in the content o representation. Retrieved August 29, 2012, from <http:// citeseerx.ist.psu.edu/viewdoc/summary?doi=10.1.1.19. 8586>.

Recibido el: 16/3/2012

Versión final el: 31/8/2012

Aprobado el: 16/10/2012 